\title{
THOMSON PARABOLA SPECTROMETER FOR ENERGETIC IONS EMITTED FROM SUB-NS LASER GENERATED PLASMAS
}

\author{
Mariapompea CutroneO ${ }^{a, b, *}$, Lorenzo Torrisi $^{a, c}$, Ludio Ando $^{c}$, \\ Salvatore Cavallaro $^{c}$, Jiri Ullschmied ${ }^{d}$, Josef Krasa $^{e}$, \\ Daniele Margarone ${ }^{e}$, Andreji Velyhan $^{e}$, Eduard Krousky $^{d, e}$, \\ Miroslav Pfeifer ${ }^{d, e}$
}

${ }^{a}$ Department of Physics, University of Messina, Messina, Italy

${ }^{b}$ CSFNSM - Centro Siciliano di Fisica Nucleare e Struttura della Materia, Catania, Italy

${ }^{c}$ INFN - Laboratori Nazionali del Sud, Catania, Italia

${ }^{d}$ Institute of Plasma Physics, ASCR, Za Slovankou 3, 18200 Prague, Czech Republic

e Institute of Physics, ASCR, Na Slovance 2, 182 21 Prague, Czech Republic

* corresponding author: m.cutroneo@tiscali.it

\begin{abstract}
Laser-generated plasmas were obtained in high vacuum by irradiating micrometric thin films ( $\mathrm{Au}, \mathrm{Au} / \mathrm{Mylar}, \mathrm{Mylar})$ with the Asterix laser at the PALS Research Infrastructure in Prague. Irradiations at the fundamental wavelength, $300 \mathrm{ps}$ pulse duration, at intensities up to about $10^{16} \mathrm{~W} / \mathrm{cm}^{2}$, enabled ions to be accelerated in forward direction with kinetic energies of the order of $2 \mathrm{MeV} /$ charge state. Protons above $2 \mathrm{MeV}$ were obtained in the direction orthogonal to the target surface in selffocusing conditions. Gold ions up to about $120 \mathrm{MeV}$ and $60^{+}$charge state were detected. Ion collectors and semiconductor $\mathrm{SiC}$ detectors were employed in time-of-flight arrangement in order to measure the ion velocities as a function of the angle around the normal direction to the target surface. A Thomson parabola spectrometer (TPS) with a multi-channel-plate detector was used to separate the different ion contributions to the charge emission in single laser shots, and to get information on the ion charge states, energy and proton acceleration. TPS experimental spectra were compared with accurate TOSCA simulations of TPS parabolas.
\end{abstract}

KEYWORDS: Thomson parabola spectrometer, high intensity laser, focal position, Opera 3D Tosca code, simulation.

\section{INTRODUCTION}

Ion acceleration driven by laser-generated plasma is a major topic in various scientific fields, from ion sources to ion implantation, nuclear physics and biomedicine.

In investigations of the macroscopic and microscopic effects occurringwhen a laser interacts with matter, one of the most important parameterd is the product $I \lambda^{2}$, where $I$ is the laser intensity and $\lambda$ is the laser wavelength. It is well known that increasing the $I \lambda^{2}$ parameter increases the ponderomotive energy transferred to plasma electrons and, consequently, the ion acceleration [10. The formation of laser plasma and its dynamics are different in cases of low or high laser power densities. In the case of low power densities, the plasma in a high vacuum chamber expands along the normal direction to the irradiated target surface with a non-relativistic velocity, and the energy of the ion beam produced is of the order of $200 \mathrm{eV}$ per charge state [9]. In the case of high densities, the ion beam expands with a relativistic velocity, the typical ion energy values being about $2 \mathrm{MeV}$ per charge state 11. The investigated ion beam must be characterized in terms of the maximum ion energy, charge states, energy distribution, etc., as well as shotto-shot reproducibility.

Various detector systems were exploited to investigate of the ion acceleration in the laser-produced plasma, one of the most useful being the Thomson parabola spectrometer (TPS). In TPS, the ions are deflected with a combined magnetic and electric field producing a mass/charge and charge state separation. Much information on the physics of ion acceleration can be obtained by analyzing the parabolas imaged on a micro-channel plate (MCP) based detector coupled to a phosphorus screen [12].

\section{Material and Methods}

At the National Laboratories in Catania, several kinds of specimens were irradiated by IR laser light from an Nd:YAG laser operating at $1 \div 10 \mathrm{~Hz}$, with an intensity of $5 \times 10^{10} \mathrm{~W} \mathrm{~cm}^{-2}, 9$ ns pulse duration and a beam diameter of $500 \mu \mathrm{m}$. It was possible to detect the plasma produced in backward direction as a consequence of thick target irradiation [6]. 
Several experiments have been carried out recently at the PALS laboratory, Prague, where an Iodine laser with $7 \times 10^{16} \mathrm{~W} \mathrm{~cm}^{-2}$ of laser intensity, $1315 \mathrm{~nm}(1 \omega)$ and $438 \mathrm{~nm}(3 \omega)$ wavelengths, $300 \mathrm{ps}$ pulse duration and a beam diameter of $70 \mu \mathrm{m}$ in single shot mode [8] has been employed for irradiating both thick and thin targets. The hottest were the forward expanding plasmas generated in interaction of the laser with thin targets. Much effort was focused on the choice of target materials, such as thick and thin polymers (polyethylene and mylar), metals ( $\mathrm{Cu}$ and $\mathrm{Au}$ ) and targets with synthesized nanoparticles.

Various tools have been used for ion detection, based on time-of-flight (TOF) techniques and on magnetic and electric ion deflections, including the Thomson parabola spectrometer.

Ion collectors (IC) were fixed at a known distance and angle from the target, and their outputs were connected to a fast storage oscilloscope. The IC current density signal depends on the ion charge, and is given by $[3$

$$
J_{\mathrm{IC}}=e z_{\mathrm{i}} n_{\mathrm{i}} v_{\mathrm{i}},
$$

where $e$ is the electron charge, $z_{\mathrm{i}}$ is the ion charge state, $n_{\mathrm{i}}$ is the ion density and $v_{\mathrm{i}}$ is the ion velocity.

At low laser intensity, the use of an ion energy analyzer (IEA) permits us to evaluate the amount of energy per charge state $(E / z)$ of the ions emitted from plasma. IEA is constituted by two coaxial metallic plates that deflect electrostatically to $90^{\circ}$ the ions arriving at a windowless electron multiplier (WEM) detector that selects them in terms of $E / z$. The ratio $E / z$ is given by the relation

$$
\frac{E}{z}=2 k V
$$

where $k$ is the gain parameter $(k=10)$ depending on the WEM and $V$ is the voltage applied to both deflection plates [7]. By varying the bias voltagei, it was possible to select different $E / z$ and finally to plot the ion energy and the charge state distributions.

$\mathrm{SiC}$ semiconductor detectors were adopted due to their fast response, their insensitivity to visible light (energy gap $3.2 \mathrm{eV}$ ) and the energy proportionality of the detected ions. Their current density signals are given by 3 .

$$
J_{\mathrm{SiC}}=e\left(n_{\mathrm{i}} \frac{E_{\mathrm{i}}}{\epsilon}\right) \mu_{\mathrm{eff}}\left(\frac{U_{\mathrm{d}}}{d}\right),
$$

where $E_{\mathrm{i}}$ is the ion energy, $\epsilon$ is the energy necessary for electron-hole pair creation, $\mu_{\text {eff }}$ is the quantum efficiencyi, which takes into account the energy loss in the detector, $U_{\mathrm{d}}$ is the bias applied to the semiconductor detector, and $d$ is the thickness of the semiconductor sensitive layer.

At high laser intensity, a Thomson parabola spectrometer was placed along the normal to the target surface in forward direction. The model of TPS employed at the PALS laboratory in Prague is shown in Fig. 1 a.



Figure 1. Model of the Thomson Parabola Spectrometer (a) Profiles of magnetic fields (b) and electric fields (c) provided by OPERA 3D/TOSCA.

Two pinholes collimate the input ions; the nearest is $1 \mathrm{~mm}$ in diameter and the other, $10 \mathrm{~cm}$ distant, is $100 \mu \mathrm{m}$ in diameter. The latter is placed at a distance of $5 \mathrm{~mm}$ with respect to the magnetic plates. The applied magnetic fields ranged between $0.05 \div 0.2 \mathrm{~T}$, and an electric voltage of $1.0 \div 3.0 \mathrm{kV}$ was applied across two deflecting plates, producing an electric field orthogonal to the direction of the incident ions.

Charged particles were deflected by electrostatic and magnetic fields towards the MCP fixed at a distance of $16.5 \mathrm{~cm}$ from the electrostatic plates. The MCP was made up with a phosphor screen $2 \mathrm{~cm}$ in diameter coupled with a CCD camera [5].

A comparison between the experimental images and the simulations carried out using Opera 3D/TOSCA code and MATLAB software [4] enabled us to evaluate the mass per charge state, the charge state and the energies of the detected ions. Figures $1 \mathrm{~b}, \mathrm{c}$ show the profiles of the magnetic and electric fields calculated using TOSCA, in order to evaluate the effects of the edge and field gradient on the ion trajectories.

\section{Results}

The measurements carried out at low laser intensities, with thick targets, provided evidence that ions are emitted from plasma in backward direction with energies of the order of $200 \mathrm{eV}$ per charge state. For Au, the maximum charge state is of the order of $10^{+}$, thus the maximum kinetic energy is of about $2 \mathrm{keV}$. The ion energy distributions follow the Coulomb-Boltzmann shifted functions, and the charge state distributions are inversely proportional to the ionization potentials of the atomic species. Investigations performed at high laser intensities, with thin targets, showed that ions emitted from plasma in the forward direction can have much higher energies, of the order of $2 \mathrm{MeV}$ per charge state. For $\mathrm{Au}$, the maximum charge state 

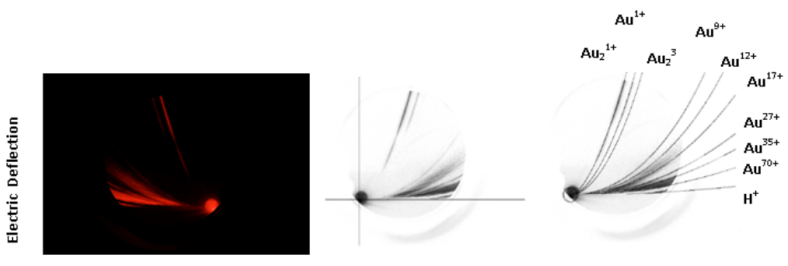

FiguRE 2. Experimental parabolas for a target of $\mathrm{Au}$ $0.6 \mu \mathrm{m}$ in thickness (a), transformation in gray scale (b) and identification of the parabolas (c).


Magnetic Deflection (mm)

FIGURE 3. Experimental parabolas obtained for targets of $\mathrm{Au}$ at three different thicknesses irradiated by an Iodine laser.

is of the order of $70^{+}$, thus the maximum kinetic energy is of about $150 \mathrm{MeV}$, as seen from the TPS spectra.

The image shown in Fig. 2 a represents a typical TPS spectrum obtained by irradiating a $0.6 \mu \mathrm{m}$ thin $\mathrm{Au}$ target. The spectrum contains a bright circular zone caused by undeflected photons and neutral particles arriving on MCP, and a lot of parabolas outgoing from this circle. Figure $2 \mathrm{p}$ shows conversion of the experimental spectrum in gray scale colors. Figure 2 c shows the simulation data overlapped with the experimental data, as obtained by Opera 3D/TOSCA code and MATLAB software. The lowermost parabola corresponds to the deflected protons, while the other parabolas corresponds to $\mathrm{Au}$ ions with high charge states.

The closer the parabola points are to the circular zone, the higher is the energy of the ions. The maximum proton energy determined from the proton parabolas was as high as $2.5 \mathrm{MeV}$. The maximum energies of $\mathrm{Au}$ ions increase with the charge state. Values of $160 \mathrm{keV}, 20 \mathrm{MeV}, 60 \mathrm{MeV}$ and $130 \mathrm{MeV}$ have been evaluated for the charge states $\mathrm{Au}^{2+}, \mathrm{Au}^{20+}$, $\mathrm{Au}^{40+}$ and $\mathrm{Au}^{60+}$, respectively. Several homogeneous $\mathrm{Au}$ samples ranging in thickness from 0.6 to $50 \mathrm{mi}-$ crons were irradiated at the same experimental and geometrical conditions for laser energy, focal position and magnetic-electric deflections.

Figure 3 features a comparison of three experimental TPS spectra obtained for different Au sample thicknesses, from 0.6 up to $50 \mu \mathrm{m}$. This experiment showed that the maximum kinetic energy of the protons emitted from laser-produced plasma in the forward direction is proportional to the target thickness. Proton energies from $2.5 \mathrm{MeV}$ ( $\mathrm{Au} 0.6 \mu \mathrm{m}$ ) up to $3.5 \mathrm{MeV}(\mathrm{Au}$ $50 \mu \mathrm{m})$ were observed.

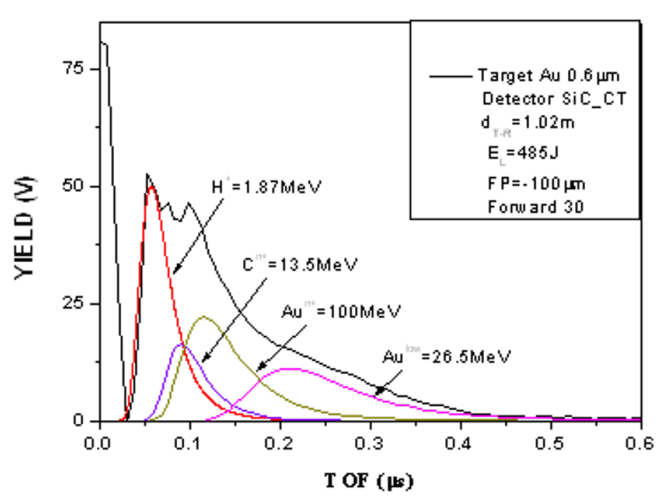

Figure 4. SiC-TOF spectrum relative to $0.6 \mathrm{~mm} \mathrm{Au}$ target irradiation.

These results can be explained on the basis of plasma electron density that increases with the thickness of the target producing enhancement of the electric field driving the ion acceleration. Of course, for thicker Au targets above $100 \mu \mathrm{m}$ the transmitted charge particles in the rear side of the target decrease so low due to the high energy loss, and practically no plasma is therefore obtainable in forward direction. A large number of forward-accelerated protons with very high kinetic energies, above $2 \mathrm{MeV}$, can be generated in high electron density plasmas produced when high-intensity laser pulses interact with thin targets made of a heavy material, such as gold.

With the aim of comparing the experimental results and the simulations, we treated the data obtained by using $\mathrm{SiC}$ detectors fixed in forward direction in a TOF approach. They agree well with TPS measurements, as seen from the $\mathrm{SiC}$ spectrum in Fig. 4. In this case, the corresponding proton peak energy is about $1.9 \mathrm{MeV}$. The proton, carbon and gold peaks can be interpreted as a convolution of the Coulomb-Boltzmann shifted distributions, according to the literature [2].

\section{Conclusions}

Thomson parabola spectrometry can provide useful detailed information on physical processes, ion species and charge states generated in single-shot laser experiments.

A combination of measurements and simulations helps to recognize well the ion species, the charge states, the maximum value of the ion energies, and the intensity distributions.

The reported results show that the energy of protons accelerated in forward direction is higher than the energy of protons accelerated in backward direction, with increasing thickness of the specimen (for micrometric thicknesses). In addition, we observed an increase in plasma temperature, with the electron density of the sample. This effect was evaluated as a first approximation from the maximum charge 
states measured through TPS. In shots with heavy metallic targets, the observed plasma temperatures and ion driving acceleration potentials were higher than those observed when using light metals or polymeric targets. The same behavior was observed for all the irradiated targets at the different laser intensities and wavelengths that were used. The results of the experiments at PALS also demonstrated well that plasma temperature, maximum kinetic ion energy and maximum charge state depend on parameter $I \lambda^{2}$, as expected [10, 11].

\section{REFERENCES}

[1] M. Borghesi, J. Fuchs, S. V. Bulanov, et al. Fast ion generation by high-intensity laser irradiation of solid targets and applications. Fusion Science and Tech 49(3):412-439, 2006.

[2] L. Laska, L. Ryc, J. Badziak, et al. Correlation of highly charged ion and x-ray emission from the laser-produced plasma in the presents of non-linear phenomena. Rad Eff \& Def in Solids 160(10-12):557-566, 2005.

[3] D. Margarone, J. Krasa, L. Giuffrida, et al. Full characterization of laser-accelerated ion beams using faraday cup, silicon carbide, and single-crystal diamond detectors. Journal of Applied Physics 109(10):103302, 2011.

[4] online. http://www.technosoft.biz/ns/tosca.php

[5] L. Torrisi, A. Borrielli, F. Caridi, et al. Optical spectroscopy in laser-generated plasma at a pulse intensity of $10^{10} \mathrm{~W} / \mathrm{cm}^{2}$. In Proc. 35th EPS-ECA, vol. 32D, p. 2.144. 2008.

[6] L. Torrisi, F. Caridi, L. Giuffrida. Protons and ion acceleration from thick targets at $10^{10} \mathrm{~W} / \mathrm{cm}^{2}$ pulse intensity. Laser and Particle Beams 29(1):29-37, 2011.

[7] L. Torrisi, M. Cutroneo. Energy analysis of protons emitted from Nd:YAG laser generated plasmas.

Radiation Effects and Defects in Solids 167(6):436-447, 2012.

[8] L. Torrisi, M. Cutroneo, S. Cavallaro, et al. Proton emission from laser-generated plasmas at different intensities. Nukleonika 57(2):237-240, 2012.

[9] L. Torrisi, L. Giuffrida, M. Rosinski, C. Schallhorn. Ge and Ti post-ion acceleration from ion source. Nucl Instr and Methods in Phys Res B 268(17-18):2808-2814, 2010 .

[10] L. Torrisi, D. Margarone, L. Laska, et al. Self-focusing effect in Au-target induced by high power pulsed laser at PALS. Laser and Particle Beams 26(3):379-387, 2008.

[11] L. Torrisi, Minniti T., L. Giuffrida. Data elaboration of proton beams produced by high-energy laser-generated plasmas. Rad Effects 63 Defects in Solids 165(6):721-729, 2010.

[12] E. Woryna, P. Parys, J. Wolowski, W. Mroz. Corpuscolar diagnostics and processing methods applied in investigation of laser-produced plasma as a source of highly ionized ions. Laser and Part Beams 14(3):293-321, 1996. 